\title{
RESERVAS DE BIOMASA DE ÁRBOLES DISPERSOS EN POTREROS Y MITIGACIÓN AL CAMBIO CLIMÁTICO ${ }^{1}$
}

\author{
Mario Chacón-León ${ }^{2}$, Celia Alice Harvey²
}

\begin{abstract}
RESUMEN
Reservas de biomasa de árboles dispersos en potreros y mitigación al cambio climático. El presente estudio tuvo como objetivo determinar la contribución de los árboles en potreros a la mitigación al cambio climático mediante la estimación de las reservas de carbono in Costa Rica y Nicaragua. Entre los años 2002 y 2003, 17783 árboles fueron inventariados en potreros de 59 fincas ubicadas en zonas de bosque tropical seco, húmedo y seco con transición a húmedo. El valor promedio de las reservas de biomasa de los árboles en los potreros fue de $10,7 \pm 1,1 \mathrm{Mg} / \mathrm{ha}$ con un valor mínimo de 5,3 $\pm 1 \mathrm{Mg} / \mathrm{ha}$ en Rivas y un máximo de 13,5 $\pm 3,5$ $\mathrm{Mg} / \mathrm{ha}$ en Matiguás. Existe relación entre la biomasa arriba del suelo de los potreros con la densidad árboles y el área basal, lo que permitió desarrollar ecuaciones simples para predecir dichos cambios. El diseño de políticas que incentiven la conservación y manejo de árboles en potreros, será un aspecto clave a ser incorporado dentro de las estrategias de cambio climático de los países.
\end{abstract}

Palabras clave: gases efecto invernadero, reservas de carbono, servicios de los ecosistemas, sistemas agroforestales, sistemas silvopastoriles.

\begin{abstract}
The contribution of dispersed trees in pastures for biomass reserve and climate change mitigation. This study aimed to explore the contribution of trees in pastures to climate change mitigation by estimating tree biomass stocks (carbon) in Costa Rica and Nicaragua. Between 2002 and 2003, 17,783 trees were inventoried in pastures of 59 farms located in areas representing three life zones: tropical dry forest, tropical wet forest and transition from tropical dry to tropical wet forest. The average value of biomass stocks of trees in pastures was $10.7 \pm 1.1 \mathrm{Mg} / \mathrm{ha}$ with a minimum value of $5.3 \pm 1 \mathrm{Mg} / \mathrm{ha}$ in Rivas and a maximum of $13.5 \pm 3.5$ $\mathrm{Mg} / \mathrm{ha}$ in Matiguás. There is a relationship between aboveground biomass in the pastureland, tree density and basal area, which allows the development of simple equations. The design of policies that encourage the conservation and management of trees in pastures will be a key aspect to be incorporated into national climate change strategies.
\end{abstract}

Key words: greenhouse gases, carbon stocks, ecosystems services, agroforestry systems, silvopastoral systems.

\section{INTRODUCCIÓN}

En América Central y alrededor del mundo, existen vastas áreas de pasturas con un alto potencial de contribuir a los esfuerzos de mitigación del cambio climático si son manejadas bajo sistemas silvopastoriles (e.j. árboles en potreros) (Jose 2009, Nair et al. 2009, Swallow y van Noordwijk 2009, FAO 2010, Thornton

\footnotetext{
1 Recibido: 19 de setiembre, 2012. Aceptado: 1 de abril, 2013. Parte del proyecto FRAGMENT (Developing methods and models for assesing the impacts of trees on farm productivity and regional biodiversity in fragmented landscapes).

2 Global Change and Ecosystem Services, Conservación International, 2011 Crystal Drive, Suite 500 Arlington, VA 22202, USA. mchacon@ conservation.org, charvey@conservation.org
} 
y Herrero 2010). Estimaciones generales proponen que el área total de pasturas en el planeta es de unas 3442 millones de hectáreas (FAO 2007) de las cuales 516 pueden estar cubiertas de sistemas agroforestales (Nair et al. 2009).

Por su parte, en América Central se estima que el área de pasturas ronda los nueve millones de hectáreas (Szott et al. 2000); muchas de estas áreas son manejadas por los finqueros en forma de sistemas silvopastoriles (Dagang y Nair 2003, Harvey et al. 2005, Harvey et al. 2010). Desafortunadamente en Centro América y otras regiones del planeta los árboles dispersos en potreros están siendo talados (Campos et al. 2001, Gibbons et al. 2008, Fischer et al. 2010) reduciendo con esto el potencial de mitigación. Para reducir la tala de árboles en potreros y entender mejor su potencial de mitigación, es necesario generar información sobre como las características de los árboles en los potreros, sobre todo a escalas locales, mitigan el cambio climático.

Actualmente existe información científica que reconoce el potencial de mitigación de los sistemas agrícolas y ganaderos (Smith et al. 2007, FAO 2010), y el tema se discute bajo la Convención Marco de las Naciones Unidas sobre Cambio Climático (Murphy y Boyle 2012). Sin embargo, la falta de información detallada sobre las cantidades de carbono presentes en forma de biomasa en sistemas silvopastoriles, hace difícil entender totalmente el rol de mitigación que juegan estos sistemas, lo cual hace aun más difícil el diseño de políticas que ayuden a conservar árboles en los potreros mientras se promueven sistemas silvopastoriles como una opción de mitigación.

El objetivo de este trabajo fue determinar la contribución de los árboles dispersos en potreros a la mitigación del cambio climático mediante la estimación de las reservas de carbono en Costa Rica y Nicaragua. Esto, con el propósito de brindar información que contribuya a diseñar prácticas silvopastoriles de manejo y que además ayuden a la creación de incentivos y políticas que promuevan el establecimiento y conservación de árboles dentro de las praderas a través del diseño de estrategias nacionales y regionales para enfrentar el cambio climático.

\section{MATERIALES Y MÉTODOS}

\section{Descripción de las áreas bajo estudio}

Este estudio se llevó a cabo en cuatro localidades agrícolas de Centro América: Cañas (entre las coordenadas $10^{\circ} 25^{\prime}$ latitud norte y $85^{\circ} 06^{\prime}$ longitud oeste, Restrepo 2002) y Río Frío (entre $10^{\circ} 22^{\prime}$ de latitud norte y $83^{\circ} 53^{\prime}$ de longitud oeste, Villacís 2003) en Costa Rica; y Matiguás (entre $85^{\circ} 27^{\prime}$ de latitud norte y $12^{\circ} 50^{\prime}$, Sauceda 2010 ) y Rivas (entre $11^{\circ} 30^{\prime}$ latitud norte y $85^{\circ} 53^{\prime}$ longitud oeste, Sánchez et al. 2004) en Nicaragua. Los cuatro sitios, de una extensión aproximada de entre 10000-16000 ha fueron seleccionados mediante el uso de imágenes satelitales y fotografías aéreas. La zonas de vida según el sistema de clasificación de Holdridge (1987), fueron Bosque Seco Tropical en Cañas y Rivas; Bosque Húmedo Tropical en Río Frío y Transición de Bosque Seco Tropical a Bosque Húmedo Tropical en Matiguás. La cobertura boscosa actual está compuesta por parches remanentes de bosques, bosques riparios y secundarios. Los sistemas de producción más importantes son ganadería de carne en Cañas, leche en Río Frío y doble propósito en Río Frío, Rivas y Matiguás (Cuadro 1).

\section{Inventario y descripción de árboles en potrero}

La recolección de datos en campo fue llevada a cabo durante febrero de 2002 y marzo de 2003 en Rivas y Cañas y desde marzo de 2003 a junio de 2004 en Río Frío y Matiguás. En cada uno de los dos sitios de Costa Rica, inventarios de árboles dispersos en potreros fueron realizados en 16 fincas, mientras que en Nicaragua se inventariaron 12 fincas en Rivas y 15 en Matiguás, para un total de 59 analizadas en este estudio. Todos los árboles dispersos en potreros con diámetro a la altura de pecho (dap) $\geq 10 \mathrm{~cm}$ fueron medidos y sus especies identificadas y registradas en una base de datos. Un total de 17783 árboles fueron registrados, representando 219 especies (211 identificadas y ocho no identificadas). Se estimó además, el promedio y el rango de la densidad de árboles en cada paisaje (número individuos/ha) y el área basal $\left(\mathrm{m}^{2}\right)$. 
Cuadro 1. Características biofísicas y productivas de las localidades en donde se realizó el estudio de estimación de reservas de biomasa de los árboles en portrero, en Cañas y Río Frío en Costa Rica; Rivas y Matiguás en Nicaragua. 2002-2003.

\begin{tabular}{|c|c|c|c|c|}
\hline Variable/Paisaje & $\begin{array}{l}\text { Cañas, } \\
\text { Costa Rica }\end{array}$ & $\begin{array}{l}\text { Río Frío, } \\
\text { Costa Rica }\end{array}$ & $\begin{array}{c}\text { Rivas, } \\
\text { Nicaragua }\end{array}$ & $\begin{array}{l}\text { Matiguás, } \\
\text { Nicaragua }\end{array}$ \\
\hline Área total (ha) & 13,051 & 15,987 & 11,621 & 10,108 \\
\hline Zona de vida según Holdridge & $\begin{array}{c}\text { Bosque Seco } \\
\text { Tropical }\end{array}$ & $\begin{array}{l}\text { Bosque Húmedo } \\
\text { Tropical }\end{array}$ & $\begin{array}{c}\text { Bosque Seco } \\
\text { Tropical }\end{array}$ & $\begin{array}{l}\text { Transición de Bos- } \\
\text { que Seco Tropical } \\
\text { a Bosque Húmedo } \\
\text { Tropical }\end{array}$ \\
\hline Altitud (m) & $80-250$ & $100-300$ & $100-200$ & $200-400$ \\
\hline Precipitación media anual (mm) & $1200-1700$ & 4120 & 1400 & 1800 \\
\hline Temperatura media $\left({ }^{\circ} \mathrm{C}\right)$ & 27,6 & 25,4 & 27,0 & 27 \\
\hline$\%$ de paisaje cubierto de pasturas & 48,4 & 47,0 & 56,7 & 68,2 \\
\hline Principal sistema productivo & Carne & $\begin{array}{l}\text { Leche (algunos de } \\
\text { carne o doble } \\
\text { propósito) }\end{array}$ & $\begin{array}{l}\text { Mixto (doble } \\
\text { propósito y } \\
\text { agricultura) }\end{array}$ & $\begin{array}{l}\text { Doble propósito } \\
\text { (algunos de } \\
\text { agricultura) }\end{array}$ \\
\hline Rango del tamaño de la finca (ha) & $5,6-1526$ & $2,5-140$ & $0,7-47,8$ & $5,62-351,3$ \\
\hline Tamaño promedio de la finca (ha) & $158,2 \pm 42,6$ & $22,1 \pm 3,1$ & $20,8 \pm 1,5$ & $27,9 \pm 5,1$ \\
\hline $\begin{array}{l}\text { Área promedio de pasturas en las } \\
\text { fincas (ha) }\end{array}$ & $47,75 \pm 42,57$ & $7,32 \pm 2,41$ & $16,05 \pm 13,11$ & $21,59 \pm 17,56$ \\
\hline $\begin{array}{l}\text { \# de fincas en donde se desarrolló el } \\
\text { inventario de árboles }\end{array}$ & 16 & 16 & 12 & 15 \\
\hline $\begin{array}{l}\text { Área total de pasturas donde se de- } \\
\text { sarrolló el inventario de árboles (ha) }\end{array}$ & 763,9 & 117,1 & 192,6 & 323,9 \\
\hline
\end{tabular}

Fuente: Harvey et al. (2010).

\section{Estimación de las reservas de biomasa arriba del suelo}

Las reservas de biomasa de los árboles arriba del suelo fueron estimadas mediante el uso de ecuaciones alométricas que estiman cantidad de biomasa seca por árbol. Para la selección de las ecuaciones apropiadas se hizo revisión de literatura y se seleccionaron las ecuaciones alométricas generales para bosques tropicales generadas por Chave et al. (2005), y para palmas por Frangi y Lugo (1985) (Cuadro 2). Se intentó aplicar ecuaciones específicas por especie de árbol, pero no se encontró una ecuación para cada especie, por lo que se procedió a utilizar una ecuación general. Estas ecuaciones requirieron el uso de las variables dap $(\mathrm{cm})$ y densidad de la madera $\left(p=\mathrm{g} / \mathrm{cm}^{3}\right)$. Se utilizaron valores específicos de densidad de la madera (Carpio 1992, Soler 2001, CATIE 2003, Soler 2004, Chave et al. 2006), lo cual fue posible para 111 especies. Donde no se encontró un valor $p$ en la revisión de literatura, se utilizó el promedio del valor $p$ por género (para 25 especies) o el valor promedio $p$ por familia (para 52 especies), según fuese el caso. Donde los valores $p$ especie, género o familia no pudieron ser obtenidos, se utilizó el valor de $0,59 \mathrm{~g} / \mathrm{cm}^{3}$, que fue el valor promedio calculado en base al valor $p$ de todas las especies presentes en este estudio.

Luego de estimar la biomasa seca arriba del suelo de cada árbol mediante las ecuaciones, se estimó mediante sumatorias las reservas de biomasa en el área total de potreros de las fincas (de cada paisaje), para posteriormente estimar por extrapolación el rango y el promedio de la biomasa por hectárea $(\mathrm{Mg} / \mathrm{ha})$. En cada localidad se crearon histogramas para resumir y comparar la distribución de las reservas de biomasa arriba del suelo según diferentes clases diamétricas y el área basal $\left(\mathrm{m}^{2}\right)$. Para identificar diferencias entre la 
Cuadro 2. Ecuaciones alométricas utilizadas para la estimación de la biomasa arriba del suelo en los árboles dispersos en potreros en Cañas y Río Frío en Costa Rica, y Rivas y Matiguás en Nicaragua. 2002-2003.

\begin{tabular}{|c|c|c|c|c|c|}
\hline Zona de vida & $\begin{array}{c}\text { Precipitación } \\
\text { anual (mm) }\end{array}$ & Aplicada en: & Ecuación alométricas & $\begin{array}{c}\text { Rango de dap } \\
\text { (cm) }\end{array}$ & Fuente \\
\hline Bosque Seco Tropical & $1200-1700$ & Cañas, Rivas & $\begin{array}{c}\mathrm{Y}=\mathrm{p}^{*} \exp (-0,667+1,784 \\
\mathrm{en}(\mathrm{D}))+0,207(\ln (\mathrm{D}))^{2}- \\
\left.0,0281(\ln (\mathrm{D}))^{3}\right)\end{array}$ & $34-64$ & \\
\hline $\begin{array}{l}\text { Transición de Bosque } \\
\text { Seco Tropical a Bosque } \\
\text { Húmedo Tropical }\end{array}$ & $1800-3000$ & Matiguás & $\begin{array}{c}\mathrm{Y}=\mathrm{p}^{*} \exp (-1,499+2,148 \\
\ln (\mathrm{D})+0,207(\ln (\ln \mathrm{D}))^{2}- \\
\left.0,0281(\ln (\mathrm{D}))^{3}\right)\end{array}$ & $23-156$ & $\begin{array}{l}\text { Chave et al. } \\
\text { (2005) }\end{array}$ \\
\hline Bosque Húmedo Tropical & $3000-3500$ & Río Fío & $\begin{array}{c}\mathrm{Y}=\mathrm{p}^{*} \exp (-1,239+1,980 \\
\ln (\mathrm{D})+0,207(\ln (\mathrm{D}))^{2}- \\
\left.0,0281(\ln (\mathrm{D}))^{3}\right)\end{array}$ & $45-133$ & \\
\hline Palmas & & $\begin{array}{c}\text { Todos los } \\
\text { paisajes }\end{array}$ & $\mathrm{Y}=-10+6,4 * \mathrm{H}$ & & $\begin{array}{c}\text { Frangi y Lugo } \\
\text { (1985) }\end{array}$ \\
\hline
\end{tabular}

$\mathrm{Y}=$ biomasa seca $(\mathrm{kg}), \mathrm{p}=$ densidad de la madera $\left(\mathrm{g} / \mathrm{m}^{3}\right), \mathrm{D}=\mathrm{dap}, \mathrm{H}=$ altura de las palmas.

densidad de árboles (número individuos/ha), el área basal $\left(\mathrm{m}^{2}\right)$ y la biomasa por hectárea $(\mathrm{Mg} / \mathrm{ha})$ entre los paisajes, se realizaron análisis de varianza aplicando el software INFOSTAT (Di Rienzo et al. 2008).

\section{Análisis del aporte de los árboles dispersos en po- treros para la mitigación al cambio climático}

Para estudiar el potencial de los árboles dispersos en potreros para contribuir con la mitigación al cambio climático, se utilizaron regresiones lineales con variables auxiliares o "Dummy" (Rawlings et al. 1998). Esto permitió probar la relación entre la cantidad de biomasa arriba del suelo con la densidad de árboles y el área basal, reuniendo los cuatro paisajes (variables de clasificación o cualitativas) en un solo modelo de regresión. Como resultado, se desarrollaron ecuaciones para predecir cambios en la biomasa arriba del suelo por hectárea según cambio en la densidad de árboles y el área basal. Con la salvedad que el presente estudio recomienda que estas ecuaciones solo pueden ser utilizadas en sitios de América Central y México con condiciones biofísicas similares a las de este trabajo.

\section{RESULTADOS Y DISCUSIÓN}

\section{Reservas de biomasa arriba del suelo de los árboles}

El total de biomasa seca de los árboles presente en las 1397,6 ha totales de potrero inventariadas fue de 12994,3 toneladas, reservadas en un total de 17783 árboles (Cuadro 3). El valor promedio de las reservas de biomasa de los árboles por hectárea en los potreros en los cuatro paisajes fue de $10,7 \pm 1,1 \mathrm{Mg} / \mathrm{ha}$, con un valor mínimo de 5,3 $\pm 1 \mathrm{Mg} / \mathrm{ha}$ presentado en Rivas y un máximo de 13,5 $\pm 3,5 \mathrm{Mg} /$ ha en Matiguás. Los paisajes poseían reservas de biomasa estadísticamente similar, con excepción de Rivas que presentó reservas de biomasa significativamente más bajas $(\mathrm{F}=145,50$; $\mathrm{p}<0,0001)$.

Los resultados mostrados previamente son similares a reportes provenientes de Centro América en donde para zonas de vida de bosque húmedo se han estimado valores de 3,2 a 14,2 Mg/ha en árboles dispersos en potreros en Esparza, Costa Rica y de 9 a 17,9 $\mathrm{Mg} /$ ha en Matiguás, Nicaragua (Ibrahim et al. 2007). En zonas de clima seco en México como la región de los Tuxtlas se han reportado valores promedios de 
Cuadro 3. Características y biomasa arriba del suelo de los árboles dispersos en potreros en cuatro localidades de Centro América, Cañas y Río Frío en Costa Rica; Rivas y Matiguás en Nicaragua. 2002-2003.

\begin{tabular}{|c|c|c|c|c|c|}
\hline Características & $\begin{array}{c}\text { Cañas, } \\
\text { Costa Rica }\end{array}$ & $\begin{array}{l}\text { Río Frío, } \\
\text { Costa Rica }\end{array}$ & $\begin{array}{c}\text { Rivas, } \\
\text { Nicaragua }\end{array}$ & $\begin{array}{l}\text { Matiguás, } \\
\text { Nicaragua }\end{array}$ & $\begin{array}{l}\text { Para los cua- } \\
\text { tro paisajes }\end{array}$ \\
\hline \# de fincas donde se realizó el inventario & 16 & 16 & 12 & 15 & 59 \\
\hline \# total de árboles registrados & 5831 & 2481 & 2290 & 7181 & 17783,0 \\
\hline Total biomasa arriba del suelo en el paisaje (Mg) & 7818,3 & 1459,6 & 802,1 & 2914,4 & 12994,3 \\
\hline Rango de la biomasa arriba del suelo ( $\mathrm{Mg} / \mathrm{ha})$ & $3,0-23,3$ & $3,4-25,7$ & $0,9-19,5$ & $2,8-45,4$ & $0,9-45,4$ \\
\hline Promedio de la biomasa arriba del suelo $(\mathrm{Mg} / \mathrm{ha})^{*}$ & $10,2 \pm 1,3 \mathrm{a}$ & $12,4 \pm 1,0 \mathrm{a}$ & $5,3 \pm 1,5 b$ & $13,5 \pm 3,5 \mathrm{a}$ & $10,7 \pm 1,1$ \\
\hline $\begin{array}{l}\text { Rango de la densidad de árboles en potreros } \\
\text { (individuos arboles/ha) }\end{array}$ & $3,1-16,9$ & $7,3-44,6$ & $2,7-67,0$ & $4,2-74,5$ & $2,7-74,5$ \\
\hline $\begin{array}{l}\text { Promedio densidad de árboles en potreros } \\
\text { (árboles/ha) }\end{array}$ & $7,8 \pm 1,0 \mathrm{c}$ & $23,1 \pm 3,0 \mathrm{ab}$ & $16,8 \pm 5,1 \mathrm{bc}$ & $30,1 \pm 5,7 \mathrm{a}$ & $19,5 \pm 2,2$ \\
\hline Área basal total en el paisaje $\left(\mathrm{m}^{2}\right)$ & 1208,6 & 300,3 & 142,7 & 296,4 & 1948.0 \\
\hline Rango del área basal $\left(\mathrm{m}^{2} / \mathrm{ha}\right)$ & $0,5-3,2$ & $0,8-4,5$ & $0,2-3,4$ & $0.3-3,7$ & $0,2-4,5$ \\
\hline Promedio área basal $\left(\mathrm{m}^{2} / \mathrm{ha}\right)$ & $1,6 \pm 0,2 b$ & $2,6 \pm 0,3 a$ & $1,0 \pm 0,2 \mathrm{~b}$ & $1.3 \pm 0,3 b$ & $1,7 \pm 0,2$ \\
\hline
\end{tabular}

Promedios en la misma línea con la misma letra no presentan diferencias estadísticas según análisis de varianza aplicando el método de Fisher-LSD, p<0,001.

* Se aplicó un ANOVA bajo un marco de modelos mixtos para considerar heterogeneidad de las varianzas. La exactitud del modelo seleccionado fue hecho con medias de AIC y BIC y "likelihood ratio test”.

biomasa de árboles en potrero de $13 \mathrm{Mg} / \mathrm{ha}$ (Hughes et al. 2000) y en Jalisco de 5,9 a 7,7 Mg/ha (Jaramillo et al. 2003).

\section{Biomasa arriba del suelo según distribución diamé- trica de los árboles}

En los cuatro paisajes se encontró como patrón general, que las mayores cantidades de biomasa la aportaban los árboles dentro de la clase mayor a 60 $\mathrm{cm}$, que a su vez fue la clase diamétrica en dónde se encontraron menos individuos de árboles, en comparación con las otras clases (Figura 1).

Otro patrón compartido por los paisajes, con excepción de Cañas en Costa Rica, fue que la mayoría de los árboles se agruparon en la clase diamétrica de entre 10 a $19 \mathrm{~cm}$; es importante tomar esto en consideración, puesto que es claro que los árboles de gran tamaño mostraron las mayores cantidades de biomasa en los paisajes, mientras que los de menor tamaño (que reservaron menos biomasa y que fueron la gran mayoría presente en los paisajes estudiados), pueden tener un alto potencial de remoción de carbono atmosférico durante su crecimiento, siempre y cuando estos sean manejados adecuadamente. Estudios realizados en Costa Rica (Jobse 2008) también reconocen la importancia que tienen los árboles de mayor tamaño para conservar reservas de carbono en potreros, recalcando la necesidad de proteger estos árboles.

\section{Potencial de los árboles dispersos en potreros para incrementar las reservas de biomasa}

En este estudio se mostró que a mayor cantidad de árboles (árboles/ha) y a mayor área basal $\left(\mathrm{m}^{2} / \mathrm{ha}\right)$, mayor será la cantidad de reservas de biomasa arriba del suelo en los potreros ( $\mathrm{Mg} / \mathrm{ha})$, lo cual fue demostrado mediante las pruebas de regresión lineal realizadas.

En los cuatro paisajes, las pruebas de regresión lineal, mostraron que existió relación entre la biomasa de los árboles con la densidad de árboles y el área basal, cuando estas variables fueron probadas en conjunto 

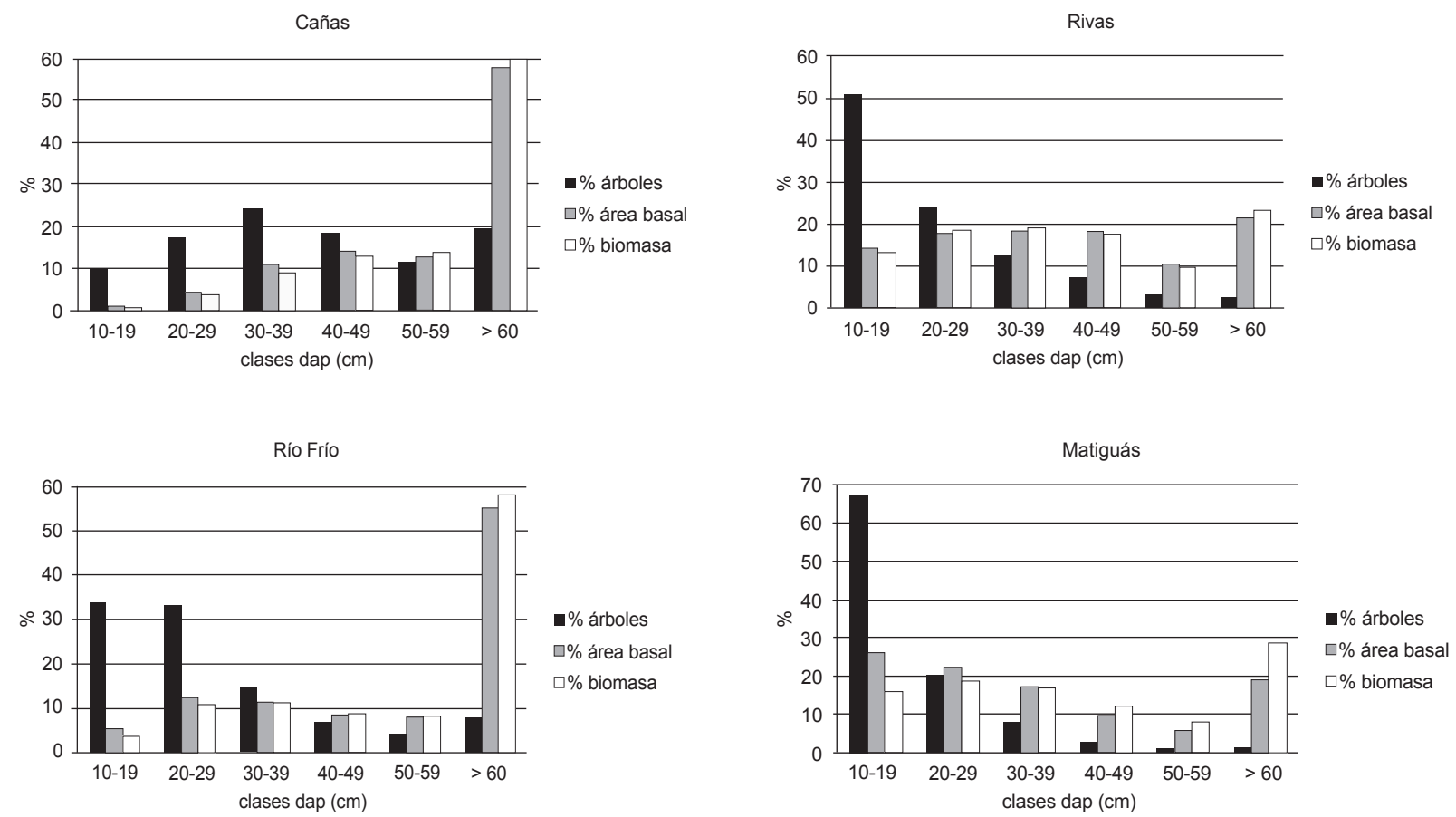

Figura 1. Porcentaje de árboles, área basal y biomasa, presente en potreros de Cañas y Río Frío en Costa Rica; Rivas y Matiguás en Nicaragua. 2002-2003.

dap: diámetro a la altura del pecho.

$\left(R^{2}=0,86, p<0,0001\right)$. Por otra parte, cuando se buscó la relación solo entre biomasa y densidad de árboles, esta fue menor pero aún siendo estadísticamente significativa $\left(\mathrm{R}^{2}=0,69, \mathrm{p}<0,0001\right)$, mientras que la relación de la biomasa con el área basal de los árboles fue más estrecha $\left(R^{2}=0,98, p<0,0001\right)$.

Basados en los resultados, se desarrollaron ecuaciones para la predicción del cambio en el contenido de biomasa según el número de árboles y el área basal (Cuadro 4). Estas ecuaciones pueden utilizarse para realizar estimaciones preliminares sobre reservas de biomasa arriba del suelo de árboles en potreros, o para estimar cambios en la biomasa cuando la densidad de árboles y/o el área basal de los árboles cambian.

Los resultados previamente descritos refuerzan aquellos presentados por estudios previos (Hughes et al. 2000, Jaramillo et al. 2003, Ibrahim et al. 2007, Jobse 2008) sobre la importancia de las reservas de biomasa en los sistemas silvopastoriles y además pre- sentan datos valiosos que pueden ayudar a mejorar el entendimiento del potencial de mitigación de los árboles en potreros en regiones que se encuentren desde el sur de México y toda la región centroamericana.

Los árboles dispersos e identificados en los potreros son elementos comunes en América Central, México y Panamá. Muchos de esos sistemas silvopastoriles son dominados por las mismas especies que las presentes en este estudio, siendo algunas de las más comunes: Cordia alliodora, Guazuma ulmifolia, Tabebuia rosea y Enterolobium cyclocarpum (Love y Spaner 2005, Zomer et al. 2009, Harvey et al. 2010, Soto-Pinto et al. 2010, Grande et al. 2010). Dada la similaridad de la composición de los sistemas silvopastoriles en la región centroamericana, la información obtenida permite la realización de estimaciones y preveer cambios en las reservas de biomasa de los potreros considerando el número de árboles presentes y la medida de su área basal. 
Cuadro 4. Ecuaciones para la estimación de biomasa de árboles dispersos en potreros $(\mathrm{Mg} / \mathrm{ha})$ en cuatro localidades en América Central en potreros de Cañas y Río Frío en Costa Rica; Rivas y Matiguás en Nicaragua, según densidad de árboles y área basal. 2002-2003.

\begin{tabular}{lllc}
\hline Paisaje & Zona de vida & Ecuación & Adj R \\
\hline General & & $\mathrm{Y}=2,9+5,05(B A)-0,16(\mathrm{~N})+0,0041\left(N^{2}\right)$ & 0,86 \\
& & Se requiere solo número de individuos arboles/ha \\
Cañas & Bosque Seco Tropical & $\mathrm{Y}=1,66+1,09(\mathrm{~N})$ & 0,69 \\
Río Frío & Bosque Húmedo Tropical & $\mathrm{Y}=9,63+0,12(\mathrm{~N})$ & \\
Rivas & Bosque Seco Tropical & $\mathrm{Y}=0,57+0,02(\mathrm{~N})$ & \\
Matiguás & Transición de Bosque Seco Tropi- & $\mathrm{Y}=-2,75+0,54(\mathrm{~N})$ & \\
& cal a Bosque Húmedo Tropical & & \\
& & $\mathrm{Se}$ requiere solo área basal & \\
Cañas & Bosque Seco Tropical & $\mathrm{Y}=-0,77+7,06(\mathrm{BA})$ & \\
Río Frío & Bosque Húmedo Tropical & $\mathrm{Y}=-1,99+5,64(\mathrm{BA})$ & \\
Rivas & Bosque Seco Tropical & $\mathrm{Y}=-0,23+5,73(\mathrm{BA})$ & \\
Matiguás & Transición de Bosque Seco Tropi- & $\mathrm{Y}=-1,57+11,36(\mathrm{BA})$ & \\
& cal a Bosque Húmedo Tropical & & \\
\hline
\end{tabular}

La ecuación es resultado de pruebas de regresión lineal con significancia estadísticas $\mathrm{p}<0,0001$. Y=biomasa Mg/ha, $\mathrm{N}=$ número de árboles/ha, BA=área basal $\mathrm{m}^{2} / \mathrm{ha}$.

Se debe recalcar la importancia de conservar árboles de mayor tamaño y de incrementar la densidad de árboles en potreros, con el fin de potencializar más aún la mitigación al cambio climático. Para lograr esto, los desarrolladores de proyectos y los gobiernos se enfrentan ante la situación de que los árboles en potreros están siendo extraídos para obtener madera y leña (Barrantes y Salazar 2008); y por otro lado, los dueños de las fincas a veces son recios a incorporar árboles en potreros, pues muchas veces esta actividad es percibida como una actividad difícil de poner en práctica y que puede incrementar costos de producción (Dagang y Nair 2003). La creación de políticas y estrategias de mitigación al cambio climático que busquen proteger e incrementar la densidad de árboles dispersos en potreros, será un paso crítico y necesario.

Los esquemas de pago por servicios de los ecosistemas, pueden ser una alternativa que permita que los sistemas silvopastoriles sean económicamente atractivos para los finqueros; sin embargo, aún existe la necesidad de reducir costos de transacción relacionados con evaluaciones de campo y monitoreo (Alarcon et al. 2001, Kelsey et al. 2008). Las ecuaciones de estimación de cambio en biomasa según densidad de árboles y/o área basal desarrolladas en este estudio, pueden ayudar a reducir el costo de estimación de biomasa necesario para mediciones y monitoreo.

La incorporación de árboles dispersos en potreros (a través de sistemas silvopastoriles) tiene un alto potencial de conservar e incrementar las reservas de biomasa y carbono en paisajes de México y América Central en donde las pasturas sean el uso de la tierra predominante. Lamentablemente, este potencial podría quedar en riesgo si los productores siguen cortando los árboles y si las políticas de los países no incentivan o reconocen la importancia de estos, tanto para la mitigación y adaptación al cambio climático (Verchot et al. 2007, Manning et al. 2009), como también para la conservación de biodiversidad y la provisión de servicios ambientales (Jose 2009, Harvey et al. 2010). Es importante diseñar nuevos proyectos de investigación que permitan el desarrollo de ecuaciones alométricas para la determinación de biomasa por especie de árbol creciendo en potreros. 


\section{AGRADECIMIENTOS}

A Fernando Casanoves, Jefe de la Unidad de Bioestadística del Centro Agronómico de Investigación y Enseñanza (CATIE) por el apoyo en los análisis estadísticos. A Hugo Brenes por el apoyo en el manejo de la base de datos.

Esta investigación se realizó como parte del proyecto FRAGMENT (Developing Methods and Models for Assessing the Impacts of Trees on Farm Productivity and Regional Biodiversity in Fragmented Landscapes), financiado por el European Community Fifth Framework Programme (INCO-Dev ICA4CT-2001-10099).

\section{LITERATURA CITADA}

Alarcon, L; Díaz, O; Dimas, L; Gonzáles, M; Herrador, M; Segura, E. 2001. Costos de prácticas agrícolas para la generación de Servicios Ambientales en El Salvador. Trabajo realizado conjuntamente por PRISMA y el Centro Nacional de Tecnología Agropecuaria y Forestal. El Salvador. 27 p.

Barrantes, A; Salazar, G. 2008. Usos y aportes de la madera en Costa Rica Estadísticas 2007. Oficina Nacional Forestal. 32 p.

Campos, JJ; Villalobos, R; Camacho, M; Gómez, M; Rodríguez, CM. 2001. La tala ilegal en Costa Rica: un análisis para la discusión. Informe elaborado por el Centro Agronómico Tropical de Investigación y Enseñanza (CATIE) a solicitud de la Comisión de Seguimiento del Plan Nacional de Desarrollo Forestal. Turrialba, Costa Rica. 53 p.

Carpio, IM. 1992. Maderas de Costa Rica 150 especies forestales. Editorial Universidad de Costa Rica. San José, Costa Rica. 350 p.

CATIE (Centro Agronómico Tropical de Investigación y Enseñanza, CR). 2003. Árboles de Centroamérica: un manual para extensionistas. CATIE. Turrialba, CR. $1079 \mathrm{p}$.

Chave, J; Andalo, C; Brown, S; Cairn, MA; Chambers, JQ; Eamus, D; Folster, H; Fromard, F; Higuchi, N; Kira, T; Lescure, J; Nelson, BW; Ogawa, H; Puig, H; Riera B;Yamakura, T. 2005. Tree allometry and improved estimation of carbon stock and balance in tropical forests. Oecologia 145:87-99.

Chave, J; Muller-Landau, HC; Baker, TR; Easdale, TA; Ter Steege,H; Webb, CO. 2006. Regional and phylogenetic variation of wood density across 2456 neotropical tree Species. Ecological Applications 16(6):2356-2367.

Dagang, ABK; Nair, PKR. 2003. Silvopastoral research and adoption in Central America: recent findings and recommendations for future directions. Agroforestry Systems 59:149-155.

Di Rienzo, JA; Casanoves, F; Balzarini, MG; Gonzalez, L; Tablada, M; Robledo, CW. 2008. InfoStat version 2008. InfoStat Group, College of Agricultural Sciences, National University of Córdoba, Argentina (en línea). Consultado enero 2011. Disponible en: http:// www.infostat.com.ar

FAO (Food and Agriculture Organization of the United Nations). 2007: State of the world's forests 2007 (en línea). Consultado noviembre 2011. Disponible en http://www.fao.org/docrep/009/a0773e/a0773e00.htm

FAO (Food and Agriculture Organization of the United Nations). 2010. Challenges and opportunities for carbon sequestration in grassland systems. A technical report on grassland management and climate change mitigation. Integrated Crop Management vol $9.57 \mathrm{p}$.

Fischer, J; Zerger, A; Gibbons, P; Stott, J; Law, BS. 2010. Tree decline and the future of Australian farmland biodiversity. PNAS 107(45):19597-19602.

Frangi, JL; Lugo, AE. 1985. Ecosystem dynamics of a subtropical floodplain forest. Ecological Monographs 55:351-369.

Gibbons, P; Lindenmayer, DB; Fischer, J; Manning, AD; Weinberg, A; Seddon, J; Ryan, P; Barrett, G. 2008. The future of scattered trees in sgricultural landscapes. Conservation Biology 22(5):1309-1319.

Grande, D; de Leon, F; Nahed, J; Perez-Gil, F. 2010. Importance and function of scattered trees in pastures in the Sierra Region of Tabasco México. Research Journal of Biological Sciences 5(1):75-87.

Harvey, CA;Villanueva, C; Villacís, J; Chacón, M; Muñoz, D; López, M; Ibrahim, M; Taylor, R; Martínez, JL; Navas, A; Sáenz, J; Sánchez, D; Medina, A; Vílchez, S; Hernández, B; Pérez, A; Ruiz, F; López, F; Lang, I; Kunth, S; Sinclair, FL. 2005. Contribution of livefences to the ecological integrity of agricultural landscapes. Agriculture, Ecosystems and Environment 111:200-230.

Harvey, CA; Villanueva, C; Esquivel, H; Gómez, R; Ibrahim, M; López, M; Martínez, J; Muños, D; Claudia, R; Saénz, J; Villacis, J; Sinclaier, F. 2010. Conservation value of dispersed tree cover threatened by pasture management. Forest Ecology and Management 261:1664-1674.

AGRONOMÍA MESOAMERICANA 24(1):17-26. 2013 
Holdridge, LR. 1987. Ecología basada en zonas de vida. Trad. Humberto Jiménez Saa. IICA. San José, Costa Rica. 216 p.

Hughes, F; Kauffman, JB; Jaramillo, VJ. 2000. Ecosystemscale impacts of deforestation and land use in a humid tropical region of Mexico. Ecological Applications 10(2):515-527.

Ibrahim, M; Chacón, M; Cuartas, C; Naranjo, J; Ponce, G; Vega, P; Casasola, F; Rojas, J. 2007. Almacenamiento de carbono en el suelo y la biomasa arbórea en sistemas de usos de la tierra en paisajes ganaderos de Colombia, Costa Rica y Nicaragua. Agroforestería en las Américas 45:27-36.

Jaramillo, VJ; Kauffman, JB; Rentería-Rodriguez, L; Cummings, DL; Elingson, LJ. 2003. Biomass, carbon, and nitrogen pools in Mexican Tropical Dry Forest Landscapes. Ecosystems 6:609-629.

Jobse, CJ. 2008. Impacts of forest-to-agriculture conversion on aboveground and soil carbon and nitrogen stocks along a bioclimatic gradient in Costa Rica. Tesis $\mathrm{PhD}$ Oregon State University. USA. 186 p.

Jose, S. 2009. Agroforestry for ecosystem services and environmental benefits: an overview. Agroforest Systems 76:1-10.

Kelsey, J; Kousky, C; Sims, KRE. 2008. Designing payments for ecosystem services: Lessons from previous experience with incentive-based mechanisms. PNAS 105(28):9465-9470.

Love, B; Spaner, D. 2005. A survey of small-scale farmers using trees in pastures in Herrera Province, Panama. Journal of Sustainable Forestry 20(3):37-65.

Manning, AD; Gibbons, P; Lindenmayer, DB. 2009. Scattered trees: a complementary strategy for facilitating adaptive responses to climate change in modified landscapes? Journal of Applied Ecology 6:915-919.

Murphy, D; Boyle, J. 2012. Agriculture and climate change: Post-Durban issues for negotiators. IISD Food Security and Climate Change Initiative. The International Institute for Sustainable Development (IISD). Canada. 13 p. (en línea). Consultado junio 2012. Disponible en http://www.iisd.org/publications/pub.aspx?id=1604

Nair, PKR; Kumar, BM; Nair, VD. 2009. Agroforestry as a strategy for carbon sequestration. Journal of Plant Nutrition and Soil Science 172:10-23.

Rawlings, JO; Pantula, SG; Dickey, DA. 1998. Applied regression analysis: A research tool. 2 ed. SpringerVerlag, New York, Inc. USA. 659 p.

Restrepo, C. 2002. Relaciones entre la cobertura arbórea en potreros y la producción bovina en fincas ganaderas en el trópico seco, Cañas, Costa Rica. Tesis de maestría. CATIE. Turrialba, Costa Rica. 102 p.

Sauceda, M. 2010. Impacto de arreglo espacial del componente arbóreo en sistemas silvopastoriles sobre el nivel de sombreado y conectividad estructural de los paisajes de Belén y Matiguás, Nicaragua. Tesis, CATIE, Turrialba, Costa Rica. 110 p.

Sánchez, D; López, M; Medina, M; Gómez, G; Harvey, CA; Vilchez, S; Hernández, B; López, F; Joya, M; Sinclair, FL; Kunth, S. 2004. Importancia ecológica y socioeconómica de la cobertura arbórea en un paisaje fragmentado de bosque seco de Belén, Rivas, Nicaragua. Encuentro 36:7-22.

Smith, P; Martino, D; Cai, Z; Gwary, D; Janzen, H; Kumar, P; McCarl, B; Ogle, S; O’Mara, F; Rice, C; Scholes, B; Sirotenko, O. 2007. Agriculture. In Metz, B; Davidson, OR; Bosch, PR; Dave, R; Meyer, LA. eds. Climate change 2007: Mitigation. Contribution of working group III to the fourth assessment report of the intergovernmental panel on climate change. Cambridge University Press, Cambridge, United Kingdom and New York, NY, USA. p. 497-540.

Soler, M. 2001. Mil maderas I. Editorial UPV (Universidad Politécnica de Valencia), Valencia. España. 598 p.

Soler, M. 2004. Mil maderas II. Editorial UPV (Universidad Politécnica de Valencia), Valencia. España. 604 p.

Soto-Pinto, L; Anzueto, M; Mendoza, J; Jimenez, G; de Jong, B. 2010. Carbon sequestration through agroforestry in indigenous communities of Chiapas, Mexico. Agroforest Syst 78:39-51.

Swallow, BM; van Noordwijk, M. 2009. Direct and indirect mitigation through tree and soil management. IFPRI 2020 Focus 16 Brief 4. International Food Policy Research Institute. Washington DC, USA. 2 p.

Szott, L; Ibrahim, M; Beer, J. 2000. The hamburger connection hangover: cattle, pasture land degradation and alternative land use in Central America, CATIE-DANIDA-GTZ, Turrialba, Costa Rica. Serie técnica, Informe técnico No. 313. CATIE, Turrialba, Costa Rica. 133 p.

Thornton, PK; Herrero, M. 2010. Potential for reduced methane and carbon dioxide emissions from livestock and pasture management in the tropics. Proceedings of the National Academy of Sciences 107(46):19667-19672.

Verchot, LV; Van Noordwijk, M; Kandji, S; Tomich, T; Ong, C; Albrecht, A; Mackensen, J; Bantilan, C; Anupama, KV; Palm, C. 2007. Climate change: linking adaptation and mitigation through agroforestry. Mitig Adapt Strat Glob Change 12:90-918.

AGRONOMÍA MESOAMERICANA 24(1):17-26. 2013 
Villacís, J. 2003. Relaciones entre la cobertura arbórea y el nivel de intensificación de las fincas ganaderas en Río Frío, Costa Rica. M.Sc. Tesis, CATIE, Turrialba, Costa Rica. 129 p.
Zomer, JR; Trabucco, A; Coe, R; Place, F. 2009. Trees on farm: analysis of global extent and geographical patterns of agroforestry. ICRAF Working Paper no. 89. Nairobi, Kenya: World Agroforestry Centre. 63 p. 